\title{
Short Term Outcome of Haemorrhagic Shock in Trauma at Mulago Hospital, an Urban Tertiary Hospital in Sub Saharan Africa
}

\author{
Moses Elaju ${ }^{1}$, Peter A Ongom ${ }^{1 *}$, Stephen C Kijjambu ${ }^{1}$, Robert Wangoda ${ }^{2}$ and Patson Makobore ${ }^{1}$
}

${ }^{1}$ Department of Surgery, School of Medicine, Makerere University College of Health Sciences, Makerere University, P O Box 7072, Kampala, Uganda

${ }^{2}$ Department of Surgery, Mulago National Referral and Teaching Hospital, P O Box 7051, Kampala, Uganda

\begin{abstract}
Introduction: Trauma is a major public health hazard, contributing significantly to mortality. It is the leading cause of death among adolescents and young adults worldwide. Road traffic injuries come top of the list of forms of trauma in urban areas, with more than 3000 deaths occurring daily. Most deaths $(85 \%)$ occur in low and middle income countries and are a result of shock secondary to haemorrhage. There being a dearth of documented information on the short term outcome and associated factors of haemorrhagic shock in a Sub Saharan setting, a study to this effect was conducted.
\end{abstract}

Methods: A prospective descriptive cohort study was conducted from October 2012 to March 2013, involving patients attending Mulago National Referral and Teaching Hospital's Accident and Emergency Department, who had traumatic haemorrhagic shock. Patients aged 5 years and above and of both genders were included. They were managed according to the ATLS guidelines and followed-up for 24 hours. The outcomes were: the 24 hour survival or mortality of these patients; the adequacy of resuscitation of the survivors; and the distribution of factors known to affect mortality.

Results: A total of 55 participants, $40(72.7 \%)$ of them males, were recruited. The mean age was 27.2 years. There were $16(29.1 \%)$ deaths in $24 \mathrm{hrs}$. Of the survivors, $13(33.3 \%)$ of them were inadequately resuscitated when clinical signs alone were used to monitor resuscitation. Grade IV shock, time since injury, and the need for major surgery to control bleeding, contributed significantly to mortality.

Conclusion: The 24 hour mortality due to haemorrhagic shock at Mulago Hospital is slightly lower than that reported in some studies but strategies to reduce it further need to be explored.

Keywords: Haemorragic shock; Road traffic injury; Resuscitation; Grade III and IV shock; Mortality; Survivors

\section{Introduction}

Haemorrhagic shock is a life threatening condition which continues to claim the lives of patients with major trauma all over the world, but especially in middle and low income countries [1-3]. It is the leading cause of potentially preventable death among trauma patients $[4,5]$. Worldwide, trauma is the leading cause of death in children over 5 years, adolescents and young adults [6], accounting for about $10 \%$ of mortality as a whole [7]. In Uganda, a tropical Sub Saharan country, over a quarter of deaths occurring in urban areas are due to trauma $[2,8]$.

Haemorrhagic shock is defined as inadequate delivery of oxygen and nutrients to tissues due to reduced circulating volume as a result of blood loss [9-12]. It is classified into four grades: I, II, III and IV, based on the amount of blood loss and the clinical presentation of the patient. Though a life threatening condition, early recognition of haemorrhagic shock followed by timely and appropriate intervention can save lives. Management of haemorrhagic shock in a low income setting still poses a challenge due to various reasons, including lack of appropriate prehospital care and inadequate blood transfusion services.

The leading cause of haemorrhagic shock is Road Traffic Injury (RTI). The WHO report on RTI prevention (2004) estimated that 1.2 million people die worldwide in RTIs annually [1]. Of these, $85 \%$ occur in low and middle income countries. With the upsurge in the number of vehicles in low and middle income countries, mortality rate due to RTIs is anticipated to rise by $80 \%$ between 2000 and 2020 [13]. A significant number of deaths resulting from this form of trauma are due to uncontrolled haemorrhage leading to haemorrhagic shock [2]. This reflects the importance of the need for comprehensive understanding of haemorrhagic shock in order to improve patient survival. Other direct forms of trauma leading to this condition include assault, Gunshot Injury (GSI) and 'mob justice' (mob lynching), among others.

The current management of traumatic haemorrhagic shock at Mulago Hospital includes initial assessment using the ATLS guidelines; a primary survey is conducted following the Airway, Breathing, Circulation, Disability, Exposure and Environmental control (ABCDEs) approach. Identified life threatening conditions are managed, and then a secondary survey is conducted when the patient is stable. The grade of shock is also determined. In our study, we focus on grades III and IV. Grade III shock is defined as that resulting from a total blood volume loss of more than $30 \%$ but less than $40 \%$; grade IV type involves a loss of more than $40 \%$ [13]. The pulse rate and respiratory rate for

*Corresponding author: Peter A Ongom, Department of Surgery, School of Medicine, Makerere University College of Health Sciences, Makerere University, P O Box 7072, Kampala, Uganda, Tel: 256772454 936/256 701629 160; E-mail: petongom@yahoo.co.uk

Received August 17, 2013; Accepted September 09, 2013; Published September 15,2013

Citation: Elaju M, Ongom PA, Kijjambu SC, Wangoda R, Makobore P (2013) Short Term Outcome of Haemorrhagic Shock in Trauma at Mulago Hospital, an Urban Tertiary Hospital in Sub Saharan Africa. Trop Med Surg 1: 148. doi:10.4172/23299088.1000148

Copyright: (c) 2013 Elaju M, et al. This is an open-access article distributed under the terms of the Creative Commons Attribution License, which permits unrestricted use, distribution, and reproduction in any medium, provided the original author and source are credited. 
grade III shock is typically $120-140$ beats/min and $30-40$ breaths/min, respectively; the rates for grade IV shock are $>140$ beats $/ \mathrm{min}$ and $>40$ breaths/min, respectively.

Patients are transferred to the emergency surgical ward (3BES) and to the operating room for surgery when stable, or ICU for further monitoring and continued resuscitation if unstable. Definitive management of major injuries after resuscitation is done by the surgery residents and surgeons in various specialties depending on the type of the injury that caused the shock. Routine use of serum lactate as a biomarker to monitor resuscitation has not been the practice. Vital signs are relied on to determine the adequacy of resuscitation. However, it is important to consider that vital signs can be normal in the presence of ongoing tissue hypoxia.

Treatment outcome of patients with traumatic haemorrhagic shock and the distribution of factors which are known to affect the outcome were unknown at Mulago Hospital due to inadequate scientific documentation. The main objective of this study was therefore to determine the short term treatment outcome of patients with haemorrhagic shock due to trauma at Mulago Hospital and to describe the distribution of factors which are known to affect the outcome in survivors and non-survivors. It also sought to determine the adequacy of resuscitation of the survivors 24 hours after admission. Adequate resuscitation is achieved when venous serum lactate levels are $\leq 2.2$ $\mathrm{mmol} / \mathrm{L}$ at 24 hours, while inadequate resuscitation refers to levels $>2.2$ $\mathrm{mmol} / \mathrm{L}$ at 24 hours.

\section{Methods}

\section{Study design and setting}

This was a descriptive prospective study conducted among trauma patients in shock between October 2012 and March 2013. Ethical clearance was sought from Makerere University College of Health Sciences (MakCHS) Research and Ethics Committtee and Mulago Hospital's Research and Ethics Committee. It was carried out in the Accident and Emergency (A \& E) Department of Mulago National Referral and Teaching Hospital, located in Kampala, the Capital and largest city of Uganda. Kampala had an estimated population of 1,659, 600 in 2011 [14], and is a rapidly growing city. As a national referral centre it serves the whole country, as well as Eastern DRC and parts of Southern Sudan. It has a bed capacity ranging between 1,500 and 1,800 and is the main teaching hospital for Makerere University.

The hospital has an annual in-patient turnover in excess of one hundred and forty thousand $(140,000)$ patients and attends to over six hundred thousand $(600,000)$ out-patients annually. The A \& E Department has both surgical and medical wings and receives between 90,000 and 110,000 patients a year. In 2011, a total of 93,449 patients were attended to in the A \& E Department, one third of these $(31,150)$ were surgical patients. The main forms of transport in Kampala are commuter taxis (14-seater omnibuses) and "Boda-boda" taxis (local motorcycle transportation). Countrywide deaths from RTIs are on the rise, from 778 in 1990 to 2,034 in 2004.

The A \& E surgical wing has: two consultation rooms, one resuscitation room with three beds, an emergency X-ray unit, an ultrasound unit, an orthopaedic unit, a theatre with two operating rooms, a 26-bed holding emergency ward $(3 \mathrm{BE} / \mathrm{S})$, and a newly constructed Trauma Unit. The department is open $24 \mathrm{hrs}$ a day and is headed by a consultant surgeon. It is run by a variety of specialists, surgery residents, medical officers, clinical and orthopaedic officers, radiographers/sonographers and the nursing staff. Adjacent to it are the Blood Bank, Haematology, Microbiology and Clinical Chemistry laboratories. Blood products such as whole blood and packed cells are normally in short supply owing to very high demand, relatively few donors and test-kit stock outs.

On arrival at the A \& E Department, trauma patients are triaged and transferred to the examination rooms where they are attended to by doctors who institute management alongside history taking and examination. Resuscitation takes precedence throughout patient management. Patients for operative management are promptly taken to the adjacent casualty operating theatre, while those for observation and further investigations are admitted to emergency surgical ward for up to 24hrs (maximum), before onward transfer to the admitting firm or one of the specialised surgical units. On average, two patients with severe traumatic haemorrhagic shock are managed in the A \& E Department per week, giving an estimated total of 104 patients per year.

Study participants included all male and female patients aged 5 years and above, with traumatic haemorrhagic shock, who presented to the $\mathrm{A}$ \& E Department of Mulago National Hospital during the study period, met the inclusion criteria and consented/assented to participate in the study. Excluded were patients with: known liver disease (interference with lactate clearance), uncontrolled hypertension (patients may be in shock even when the blood pressure seems normal), and coexisting non-haemorrhagic causes of shock (burns and septicaemia).

A consecutive sampling method was used to obtain the required sample size. The sample size was 55 estimated using the Leslie Kish (1963) equation, with adjustments for a finite population correction for proportion to suit the study duration, and an estimated proportion of mortality due to haemorrhagic shock of 0.4 [15]. Independent variables included: grade of shock, patient age, intervention (IV fluids, blood transfusion and surgery), time to intervention, time from injury and mechanism of injury. The major outcome variables were mortality or survival, and adequacy of resuscitation.

\section{Procedure}

All trauma patients who presented to the A \& E Department were triaged by the triage nurse on arrival (Figure 1). Patients with life threatening injuries were managed first. These were taken to the resuscitation room by the support staff, caretakers or the medical personnel present at that time.

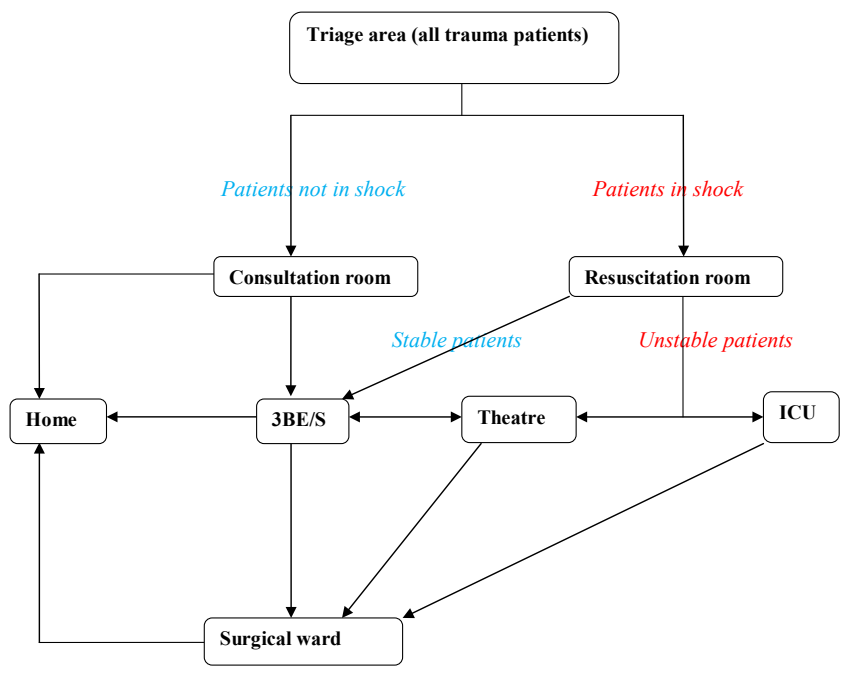

Figure 1: Patient flow diagram for A \& E Department. 
Initial assessment was carried out by an investigator (M.E) using the ATLS guidelines (Primary Survey plus resuscitation, then Secondary Survey and transfer for definitive care) [16].

Primary survey was conducted following the Airway, Breathing, Circulation, Disability and Exposure (ABCDE) approach and patients were resuscitated accordingly. Vital signs (blood pressure, pulse and respiratory rate) were taken and recorded for all trauma patients. Blood pressure (BP) and pulse rate were measured with the patients in a supine position using an electronic BP machine (Omron M2) with an appropriate cuff attached and applied to the arm 2 to $5 \mathrm{~cm}$ proximal to the ante-cubital fossa. A paediatric cuff was used to measure BP in children. Restless patients were restrained in the most appropriate manner to minimise measurement errors. Adults with a systolic $\mathrm{BP} \leq 90 \mathrm{mmHg}$ and children with systolic BP $<70+(2 \times$ Age in years $)$ were considered to be in shock and recruited into the study if they met the inclusion criteria. They were then managed according to the ATLS guidelines. Priority was given to haemorrhage control and then restoration of intravascular volume. We restricted the study to grade 3 and 4 shock. These grades are generally associated with more adverse outcomes and are better clinically defined.

The procedures were explained to the patient or the care taker, and consent obtained. An appropriate site for cannulation was identified and a tourniquet was applied about 6 to $8 \mathrm{~cm}$ proximal to it. The site was prepared with $70 \%$ isopropyl alcohol, and a cannula introduced into a vein in the standard way. Two large bore cannulae, gauge 16 for adults and a minimum of gauge 20 for children, were inserted into suitable peripheral veins and secured with tape. Six $\mathrm{mL}$ of blood were collected in a $10 \mathrm{~mL}$ syringe for haemoglobin concentration $(\mathrm{Hb})$, packed cell volume (PCV), lactate measurements, and grouping and cross-matching. Following blood sample withdrawal a giving set was connected to the appropriate IV fluid for resuscitation.

The blood was immediately transferred to vacutainers containing - EDTA for Hb and PCV; plain ones for grouping and cross matching, and sodium fluoride for lactate measurement. Samples were delivered to the laboratory as soon as possible (maximum 30 mins) during working hours, while samples collected after laboratory working hours were kept overnight in a refrigerator at $2-8^{\circ} \mathrm{C}$. Lactate was measured using the COBAS INTEGRA 400 plus machine while Complete Blood Counts (CBC) and $\mathrm{Hb}$ were measured using the NIHON KOHDEN MEK $7222 \mathrm{k}$ machine. Serum lactate was measured at admission and 24 hours post admission (for survivors).

External bleeding was controlled by applying digital pressure, pressure dressings and wound suturing. Fractured limbs were splinted and a pelvic binder was applied using any available and adequate clothing, in patients with suspected or confirmed pelvic fractures. Patients with suspected internal bleeding (intraabdominal, intrathoracic and retroperitoneal) were evaluated clinically and radiologically using Focused Assessment Sonography in Trauma (FAST) and X-Rays of the chest and pelvis, depending on the likely injury. Upon confirmation, the respective surgeons or surgery residents were contacted to review the patient and manage the bleeding. Ultimately, the mode of treatment for the patients was the prerogative of these attending doctors.

Two litres (for adults) or $20 \mathrm{~mL} / \mathrm{Kg}$ of body weight (for children), of Ringer's Lactate (RL) or Normal Saline (NS), were given as a bolus within 20 minutes. After the initial bolus, vital signs were taken in order to determine the response. If no response occurred, the initial bolus was repeated and the patient reassessed. In the event of yet no improvement after the second bolus, the patient was transfused with packed red blood cells ( 1 pint for adults; $10 \mathrm{mLs} / \mathrm{Kg}$ for children) if available or whole blood in the absence of packed red cells ( 2 pints for adults; 20 $\mathrm{mLs} / \mathrm{Kg}$ for children). Patients who needed out right urgent transfusion were transfused as soon as blood was available.

Demographic details were collected using pre-tested structured and coded interviewer administered questionnaires after the primary survey. During the secondary survey, the following were established: patient's age, mechanism of injury, time from the injury, and prehospital care given, if any. The patient's guardians and care takers were essential during this process. Any other relevant information was obtained and documented. A head to toe (including the back) examination of the patient to look for associated injuries and determine the injury severity was conducted. A urethral catheter was inserted in patients with no suspected urethral injuries, while a suprapubic one was used for those with suspected urethral injuries, in order to monitor urine output. Patients were then transferred to any of the following locations: ward 3BE/S if they became stable following resuscitation; the operation room for control of bleeding and definitive management; or the ICU if unstable, for further resuscitation and monitoring.

Patients were followed-up for 24 hours from the time of presentation to the A \& E Department. At 24 hours, vital signs for the survivors were taken again and blood samples collected for lactate measurement. The outcome of interest was evaluated at death for the non-survivors and at 24 hours post admission for the survivors.

\section{Data Analysis}

All data were entered into EpiData ${ }^{\circledR}$ version 3.1 and then transferred to Stata version 10 (StataCorp, Texas, USA) for analysis. Data were presented in tables, graphs and text. Categorical variables were summarised as frequencies and proportions, while continuous variables were summarised as means and standard deviations. Univariate and bivariate analyses were done and the $\mathrm{P}$ values were computed using the t-test for continuous variables. The Odds Ratio (OR) was used to estimate the magnitude of association or difference in distribution of the risk factors for mortality. Logistic regression was used for categorical data. Confidence intervals of $95 \%$ were used and a $P$ value $<0.05$ was considered significant. The power of the study was set at $80 \%$.

\section{Results}

A majority $(7,970)$ of the patients initially evaluated for inclusion into the study (patients attending A \& E Department) had other ailments which included traumatic and non-traumatic conditions (Figure 2). Traumatic cases refer to those who sustained injuries but were not in a state of haemorrhagic shock at admission. Non-traumatic cases included a range of surgical conditions, both emergency and elective. Elective cases were assessed and sent to the appropriate outpatient clinics. The single excluded patient was a victim of 'mob justice' who had also sustained $21 \%$ burns of the extremities and trunk besides the bleeding lacerations. There was no loss to follow-up.

Demographic and clinical characteristics are presented in Table 1. These included age, sex, time since injury and grade of shock. One participant was a known hypertensive $(\mathrm{BP}>140 / 90 \mathrm{mmHg}$ ) patient on treatment with a reportedly controlled previous BP. The Injury Severity Score (ISS) of the participants ranged from 16 to maximum. The different circumstances in which participants were injured were described as the mechanisms of injury. Road traffic crashes were the leading cause of injury (causing RTIs) (56.4\%). The 3.6\% denoted as 'Others' in the graph were accidental injuries; one patient had an industrial machine 
Citation: Elaju M, Ongom PA, Kijjambu SC, Wangoda R, Makobore P (2013) Short Term Outcome of Haemorrhagic Shock in Trauma at Mulago Hospital, an Urban Tertiary Hospital in Sub Saharan Africa. Trop Med Surg 1: 148. doi:10.4172/2329-9088.1000148

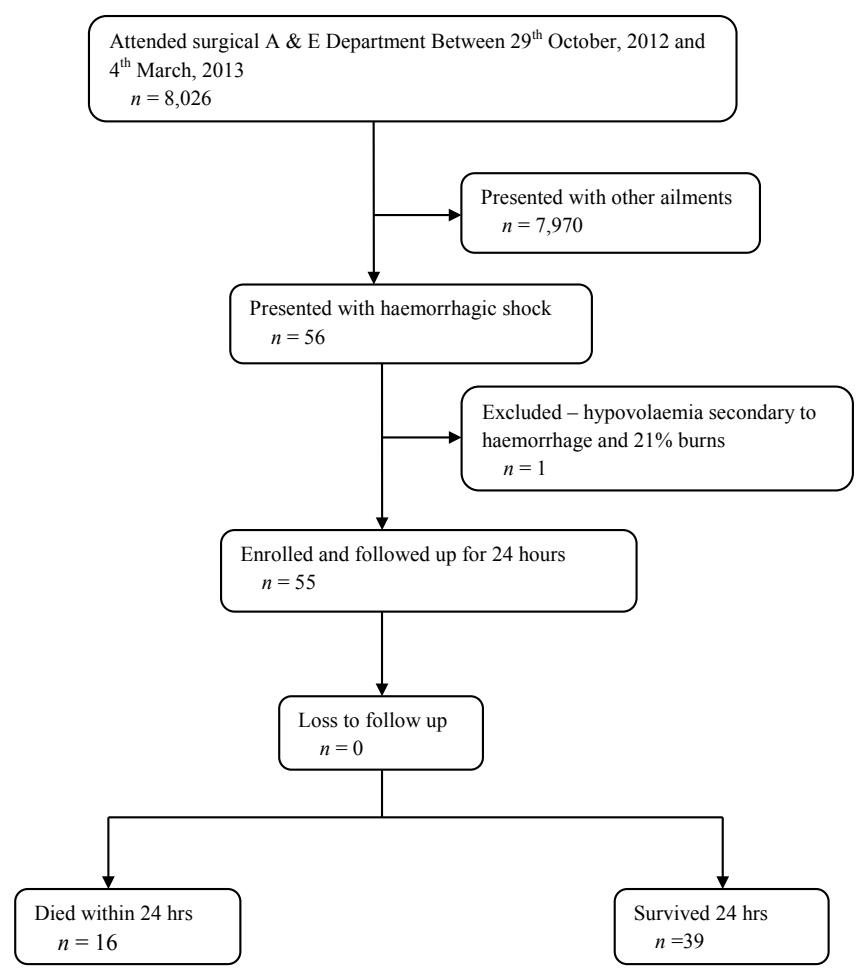

Figure 2: Study flow chart.

\begin{tabular}{|c|c|c|}
\hline \multirow{2}{*}{ Characteristic } & \multicolumn{2}{|c|}{ Distribution of study participants } \\
\cline { 2 - 3 } & Number & Percentage (\%) \\
\hline $5-12$ & & \\
\hline $13-17$ & 4 & 7.27 \\
\hline $18-40$ & 3 & 5.46 \\
\hline$>40$ & 43 & 78.18 \\
\hline Sex & 5 & 9.09 \\
\hline Female & 15 & \\
\hline Male & 40 & 27.27 \\
\hline Time since Injury & & 72.73 \\
\hline$\leq 1$ hour & 18 & \\
\hline$>1$ hour & 37 & 32.73 \\
\hline Grade of shock & & 67.27 \\
\hline Grade III & 30 & \\
\hline Grade IV & 25 & 54.55 \\
\hline
\end{tabular}

Table 1: Patient demographic and clinical characteristics

injury, while the other was cut by a piece of glass injuring the brachial artery. Injuries were also described in the context of the body region injured. The body region injured was determined by the site of the most severe injury and therefore the most likely cause of the shock (Figure 3 ). Table 2 illustrates the mechanism of injury and body region involved. Most of the extremity injuries were to the lower limbs. Specifics about the particular injury in a body region are presented in Figure 4. Scalp lacerations $(29.1 \%)$, ruptured spleen $(20.0 \%)$ and long bone fractures (12.7\%) were the dominant specific injuries. The long bone fractures included both open and closed fractures, and most involved the femoral shaft.

The distribution and type of care received by the patients is presented in Table 3. Details and particulars of the type of fluid and medication given in the pre-hospital period were difficult to ascertain. Most patients who were referred had incomplete referral forms with inadequate information. Within Mulago Hospital, patients had indications for blood transfusion and operative surgery to control haemorrage (Tables 4 and 5). The patients who did not need blood transfusion were all in grade III shock, while all patients with grade IV shock required transfusion. The overall average amount of intravenous fluids given at Mulago Hospital was $2.71 \mathrm{~L}$ (SD 1.23), while that of blood was 2.27 units (SD 1.83). The patients who were given intravenous fluids prior to admission to Mulago Hospital received on average of $2.63 \mathrm{~L}$ of fluids while in Mulago Hospital. One patient had received blood transfusion prior to admission but was given 3 more units of blood while at Mulago Hospital. Major surgery was considered to be any surgery requiring general anaesthesia, except when the general anaesthesia was given purely due to age as in children, who cannot tolerate local anaesthesia. Non major surgery methods to control bleeding included wound suture, pressure dressing and splinting for fractured limbs (Table 6).

Laboratory tests on blood yielded baseline information on $\mathrm{Hb}, \mathrm{PCV}$ and lactate levels. The population's mean $\mathrm{Hb}$ was $11.0 \mathrm{~g} / \mathrm{dL}$ (SD 3.26), The mean $\mathrm{Hb}$ of survivors was $11.3 \mathrm{~g} / \mathrm{dL}$ (SD 2.76) and that of nonsurvivors was $10.3 \mathrm{~g} / \mathrm{dL}$ (SD 4.25). The difference in mean $\mathrm{Hb}$ between survivors and non survivors was $1.1 \mathrm{~g} / \mathrm{dL}(P=0.275)$. The mean $\mathrm{Hb}$ of the patients who needed blood transfusion was $10.4 \mathrm{~g} / \mathrm{dL}$ and for those who did not need transfusion was $12.9 \mathrm{~g} / \mathrm{dL}$. The population's mean PCV was $35.3 \%$ (SD 1.22). The survivors had a mean PCV of $35.7 \%$ (SD 8.56) while the non-survivors had a mean of $34.2 \%$ (SD 2.57). The difference in mean PCV between survivors and non-survivors was $1.5 \%$ $(P=0.575)$. All 55 patients had elevated lactate levels $(>2.2 \mathrm{mmol} / \mathrm{L})$ at admission. Table 6 shows the distribution of lactate levels in relation to the mechanism of injury. Twenty four hours post admission, one third
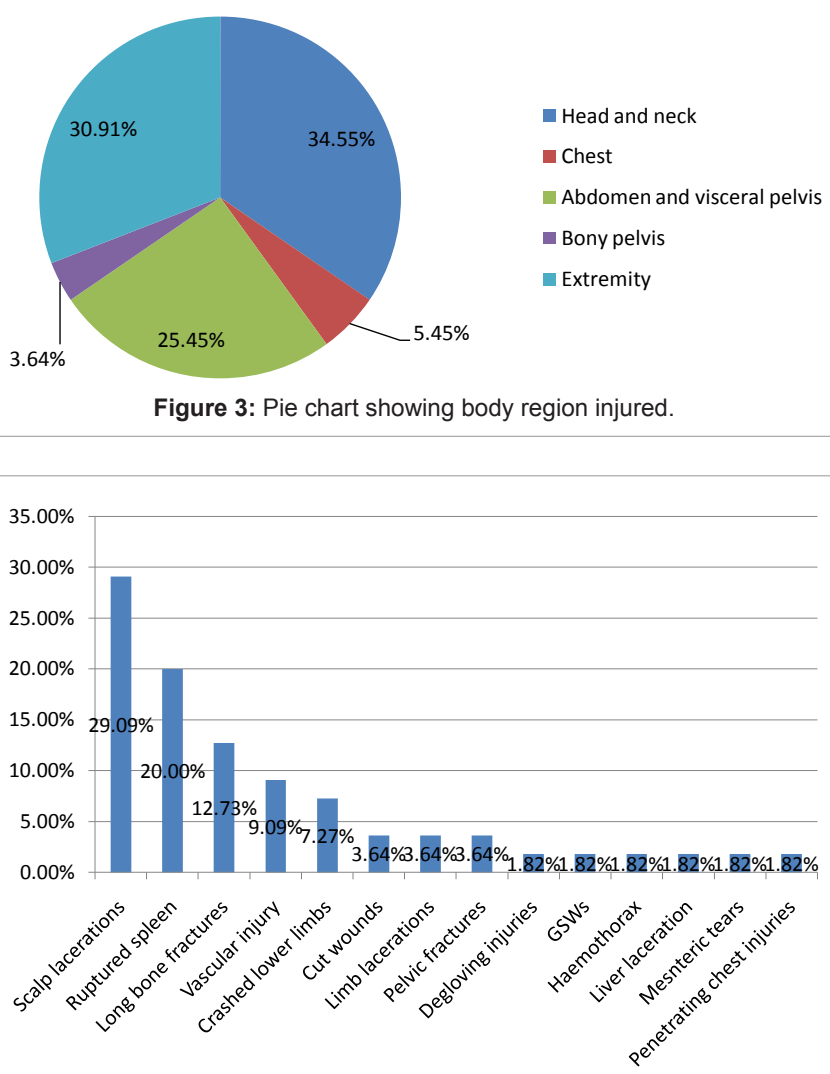

Figure 4: Bar chart showing specific type of injury sustained. 
Citation: Elaju M, Ongom PA, Kijjambu SC, Wangoda R, Makobore P (2013) Short Term Outcome of Haemorrhagic Shock in Trauma at Mulago Hospital, an Urban Tertiary Hospital in Sub Saharan Africa. Trop Med Surg 1: 148. doi:10.4172/2329-9088.1000148

Page 5 of 10

\begin{tabular}{|c|c|c|c|c|c|c|c|c|}
\hline \multirow[t]{2}{*}{ Mechanism of injury } & \multicolumn{8}{|c|}{ Body region injured, $\mathrm{n}(\%)$} \\
\hline & Head and neck & Face & Chest & $\begin{array}{c}\text { Abdomen and visceral } \\
\text { pelvis }\end{array}$ & Bony pelvis & Extremities & Total & $95 \% \mathrm{Cl}$ \\
\hline RTIs & $7(36.8)$ & $0(0.0)$ & $2(66.7)$ & $10(71.4)$ & $1(50.0)$ & $11(64.7)$ & $31(56.4)$ & $43.3-68.6$ \\
\hline Mob justice & $8(42.1)$ & $0(0.0)$ & $0(0.0)$ & $0(0.0)$ & $0(0.0)$ & $3(17.7)$ & $11(20.0)$ & $11.4-32.5$ \\
\hline Assault & $2(10.5)$ & $0(0.0)$ & $1(33.3)$ & $2(14.3)$ & $0(0.0)$ & $2(11.8)$ & $7(12.7)$ & $6.0-24.3$ \\
\hline Fall from a height & $0(0.0)$ & $0(0.0)$ & $0(0.0)$ & $2(14.3)$ & $0(0.0)$ & $0(0.0)$ & $2(3.6)$ & $0.3-13.0$ \\
\hline GSIs & $1(5.3)$ & $0(0.0)$ & $0(0.0)$ & $0(0.0)$ & $1(50.0)$ & $0(0.0)$ & $2(3.6)$ & $0.3-13.0$ \\
\hline Others & $1(5.3)$ & $0(0.0)$ & $0(0.0)$ & $0(0.0)$ & $0(0.0)$ & $1(5.9)$ & $2(3.6)$ & $0.3-13.0$ \\
\hline Total & $19(34.6)$ & $0(0.0)$ & $3(5.5)$ & $14(25.5)$ & $2(3.6)$ & $17(30.9)$ & $55(100)$ & \\
\hline
\end{tabular}

Table 2: Mechanism of injury by body region.

\begin{tabular}{|l|l|}
\hline & Number (\%) \\
\hline Distribution & \\
\hline No pre-hospital care & $47(85.45)$ \\
\hline Pre-hospital care & $8(14.55)$ \\
\hline Form of pre-hospital care & \\
\hline IV fluids & $4(50.00)$ \\
\hline Blood transfusion & $1(12.50)$ \\
\hline Medication (Unspecified) & $3(37.50)$ \\
\hline
\end{tabular}

Table 3: The distribution and form of pre-Mulago Hospital care received.

\begin{tabular}{|c|l|l|l|}
\hline & Number (\%) & Mean Hb (g/dl) & Mean PCV (\%) \\
\hline Transfusion indicated & & & \\
\hline No & $18(32.73)$ & 12.86 & 40.09 \\
\hline Yes & $37(67.27)$ & 10.42 & 32.92 \\
\hline Transfusion status & & & \\
\hline Not transfused & $7(18.92)$ & - & - \\
\hline Transfused & $30(81.08)$ & - & - \\
\hline
\end{tabular}

Table 4: Blood transfusion summary.

\begin{tabular}{|l|l|}
\hline & Number (\%) \\
\hline Major surgery indicated & \\
\hline No & $28(50.91)$ \\
\hline Yes & $27(49.09)$ \\
\hline Time to surgery & \\
\hline Within 1 hour & $4(14.81)$ \\
\hline 1-2 hours & $3(11.11)$ \\
\hline$>2$ hours & $20(74.07)$ \\
\hline
\end{tabular}

Table 5: Summary of major surgery to control bleeding.

\begin{tabular}{|l|c|c|}
\hline Mechanism of injury & $\begin{array}{c}\text { Mean serum lactate } \\
\text { (mmol/L) }\end{array}$ & Number (\%) \\
\hline RTC & 7.31 & $31(56.36)$ \\
\hline Mob justice & 7.27 & $11(20.00)$ \\
\hline Assault & 8.72 & $7(12.73)$ \\
\hline Fall from height & 4.59 & $2(3.64)$ \\
\hline Gun shot & 5.41 & $2(3.64)$ \\
\hline Others & 5.22 & $2(3.64)$ \\
\hline Total & & $55(100)$ \\
\hline
\end{tabular}

Table 6: Lactate levels at admission by mechanism of injury.

(33.3\%) of the surviving patients still had venous lactate levels $>2.2$ $\mathrm{mmol} / \mathrm{L}$, while $66.67 \%$ had normal lactate levels. Vital signs measured at $24 \mathrm{hrs}$ are presented in Table 7.

The 24 hour mortality was 16/55 (29.1\%) (Table 8). Of the patients who died, 9 were male and 7 female. Survivors had a mean age of 27.5 years (SD 10.43) and that of the non-survivors was 26.3 yrs (SD 8.33). The mean age difference between survivors and non survivors was 1.3

\begin{tabular}{|c|l|}
\hline Parameter & Distribution among survivors \\
\hline Systolic BP & Number (\%) \\
\hline$\cdot$ Normal & \\
\hline$\cdot$ Elevated & $35(98.74)$ \\
\hline Pulse rate & $4(10.26)$ \\
\hline$\cdot$ Normal & \\
\hline$\cdot$ Tachycardia & $25(64.10)$ \\
\hline Respiratory rate & $14(35.90)$ \\
\hline$\cdot$ Normal & \\
\hline$\cdot$ Tachypnoea & $20(51.28)$ \\
\hline Urine output (mLs/hr) & $19(48.72)$ \\
\hline$\cdot>30$ & $32(82.05)$ \\
\hline$\cdot 20-30$ & $7(17.95$ \\
\hline
\end{tabular}

Table 7: Vital signs and urine output at 24 hours.

\begin{tabular}{|l|l|l|l|}
\hline Outcome & Number & Percentage & 95\% Cl of percentage \\
\hline Alive & 39 & 70.91 & $(58.52-83.30)$ \\
\hline Dead & 16 & 29.09 & $(16.70-41.48)$ \\
\hline \multicolumn{4}{|c|}{ Table 8: Treatment outcome. }
\end{tabular}

yrs $(P=0.662)$. Patients who had major surgery within the first 2 hours of arrival to the A \& E Department all survived. Among those who had surgery after 2 hours, 6 survived and 14 died. The P value and OR were not applicable because those who had surgery within the first 2 hours of admission had no mortality. All the 7 patients who needed blood transfusion but did not receive it died, while only 9 of the 30 patients who received blood transfusion died. The $P$ value and the OR were not applicable since there were no survivors in the group that needed blood transfusion but did not receive it.

A number of factors affecting mortality and there distribution are illustrated in Table 9. A logistic regression analysis to determine the magnitude of association in the distribution of the known risk factors associated with death showed statistical significance for: i) the time since injury, ii) the grade of shock and iii) the need for major surgery to control bleeding.

\section{Discussion}

Fifty five patients' data were analysed at the end of the study. The 24 hours mortality was 16/55 (29.1\%). Of the factors which are known to affect mortality in patients with traumatic haemorrhagic shock, grade IV shock and the need for major surgery to control bleeding significantly increased mortality (all had $P$ values $<0.05$ ). Among survivors $33.3 \%$ were inadequately resuscitated when blood pressure measurement is used as the end point of resuscitation.

During enrolment, a majority $(7,970)$ of the patients who were screened for the study had other ailments, not leading to haemorrhagic 


\begin{tabular}{|c|c|c|c|c|}
\hline \multirow[t]{2}{*}{ Risk factors } & \multicolumn{2}{|c|}{$\begin{array}{l}\text { Distribution of patient } \\
\text { outcomes }\end{array}$} & \multirow[t]{2}{*}{ OR $(95 \% \mathrm{Cl})$} & \multirow[t]{2}{*}{$P$ value } \\
\hline & $\begin{array}{c}\text { Alive, } \\
\text { Number (\%) }\end{array}$ & $\begin{array}{c}\text { Dead, } \\
\text { Number (\%) }\end{array}$ & & \\
\hline \multicolumn{5}{|l|}{ Age categories (years) } \\
\hline $5-12$ & $3(7.69)$ & $1(6.25)$ & Reference & \\
\hline $13-17$ & $2(5.13)$ & $1(6.25)$ & $1.5(0.06-40.63)$ & 0.810 \\
\hline $18-40$ & $30(76.92)$ & $13(81.25)$ & $1.3(0.12-13.70)$ & 0.827 \\
\hline$>40$ & $4(10.26)$ & $1(6.25)$ & $0.75(0.32-17.51)$ & 0.858 \\
\hline \multicolumn{5}{|l|}{$\begin{array}{l}\text { Time from injury to } \\
\text { arrival at the A\&E } \\
\text { Department (hours) }\end{array}$} \\
\hline $1 . \leq 1$ & $9(23.08)$ & $9(56.25)$ & & \\
\hline 2. $>1$ & $30(76.92)$ & $7(43.75)$ & $0.23(0.07-0.80)$ & 0.021 \\
\hline \multicolumn{5}{|l|}{ Mechanism of Injury } \\
\hline 1. Road traffic crash & $18(46.15)$ & $13(81.25)$ & Reference & \\
\hline 2. Mob Justice & $11(28.21)$ & 0 & - & - \\
\hline 3. Assault & $5(12.82)$ & $2(12.50)$ & $0.55(0.09-3.31)$ & 0.517 \\
\hline 4. Fall from height & $2(5.13)$ & 0 & - & - \\
\hline 5. Gunshot wound & $1(2.56)$ & $1(6.25)$ & $1.38(0.08-24.23)$ & 0.824 \\
\hline 6. Others & $2(5.13)$ & 0 & - & - \\
\hline \multicolumn{5}{|l|}{ Grade of Shock } \\
\hline 1. Grade III & $29(74.36)$ & $1(6.25)$ & & \\
\hline 2. Grade IV & $10(25.64)$ & $15(93.75)$ & $43.50(5.08-372.71)$ & 0.001 \\
\hline \multicolumn{5}{|l|}{ Pre-hospital care } \\
\hline 1. No & $32(82.05)$ & $15(93.75)$ & & \\
\hline 2. Yes & $7(17.95)$ & $1(6.25)$ & $0.30(0.03-2.70)$ & 0.286 \\
\hline \multicolumn{5}{|l|}{$\begin{array}{l}\text { Need for blood } \\
\text { transfusion }\end{array}$} \\
\hline 1. No & $18(46.15)$ & 0 & & \\
\hline 2. Yes & $21(53.85)$ & $16(100)$ & - & - \\
\hline \multicolumn{5}{|l|}{ Major surgery needed } \\
\hline 1. No & $26(66.67)$ & $2(12.50)$ & & \\
\hline 2. Yes & $13(33.33)$ & $14(87.50)$ & $14(2.76-71.04)$ & 0.001 \\
\hline
\end{tabular}

Odds Ratio (OR) is used to estimate the magnitude of the association/difference in distribution of the risk factors to death

Table 9: Distribution of factors known to affect mortality.

shock. Like reported in a previous study [10], our study found that these elective and minor cases tend to congest the A \& E Department of Mulago Hospital. This is largely the result of a poor referral system and less effective peripheral health units. The presence of non-emergency conditions in the A \& E Department increases workload and interferes with the care of patients with emergency conditions and may partly be responsible for delay in surgery, to a time after one hour from admission.

\section{Demographics and patient characteristics}

The population's mean age was 27.2 years (SD 9.81), with a range of 5-51 yrs. Most of the participants were young adults between the age of 18 and 40 years. RTIs were the leading cause of injury in all the age groups. Assault and mob justice were also more common in the same age group. Children and adolescents were less likely to be injured by assaults and mob justice. They spend most of their time either at home or at school, a relatively less risky environment for injuries. Adults over 40 years of age are also at less risk probably because of the characteristically cautious nature of the middle-aged and elderly, as compared to young adults. There was no significant difference in mean age between survivors and non-survivors $(P=0.662)$. This is likely to be due to similarity in age distribution between survivors and nonsurvivors. Age as a factor is known to affect the outcome of traumatic haemorrhagic shock mainly in elderly patients who cannot mount an appropriate response to shock due to deficiency in catecholamine receptors [17].

As reported in other studies $[12,18,19]$, this one points out the tendency of traumatic injuries and their consequences to occur in the young population. This is possibly because young people are more physically active compared to older ones. The finding has socioeconomic implications at household and national levels since young people are the most productive age group [18]. An upsurge in the number of vehicles, lack of protective gear, poor traffic control, reckless driving/riding and a poor road infrastructure could all explain why road traffic crashes were the leading cause of injury in all the age groups. The frequent occurrence of mob justice among young adults could be due to the high unemployment rates, and increase in crime and lawlessness. Children and adolescents were less likely to be injured by mob justice and assaults ostensibly because they do not often engage in criminal and lawless activities. Most of them are dependants and are not directly affected by unemployment. The study population had 40 (72.3\%) males and 15 (27.3\%) females, giving a male to female ratio of 2.7:1. Male predominance in this study is consistent with findings of other trauma related studies $[12,18,19]$. Despite this predominance, there was no significant difference in mortality between males and females in this study $(P=0.082)$.

The distribution of specific injuries between males and females was remarkably different. Ruptured spleen, vascular injuries and long bone fractures were commoner in males than females. All the scalp lacerations were in males. Crash injuries of the lower limbs on the other hand were commoner in females than males, and tended to be bilateral. The tendency of bilateral crash injuries of the lower limbs to occur in females could be related to their sideways sitting position while on 'boda-bodas' (motorcycle taxis). Both limbs are exposed to the crashing agent, unlike in males who tend to sit astride. The lack of significance in mortality between males and females could possibly have been due to the difference in the type of injuries sustained. Whereas males were generally more involved than females, this did not translate into higher mortality. A possible explanation could be because the type of injuries sustained by males such as scalp lacerations and long bone fractures were amenable to haemorrhage control by external compression using digital pressure, compression dressing, wound suturing and splinting of fractured limbs.

\section{Clinical characteristics}

RTIs still remain the leading cause of injuries (56.4\%), a finding which is consistent with that of other trauma related studies $[17,18]$. There are observed associations between the mechanism of injury and the body region injured (Table 2). Most of the injuries due to RTIs were to the abdomen, extremities, and head and neck regions.

During a road traffic crash, bumper injuries tend to affect the lower extremities in adult pedestrians, and the abdomen in children. However, the abdomen in adults may be injured when bigger vehicles are involved in the crash. 'Boda-boda' injuries on the other hand tend to affect the extremities and head. Lack of protective gear, helmets at that, is implicated in head and neck injuries especially in 'boda-boda' crashes. The above factors may explain the pattern of injuries seen in Table 2. The victims of mob justice and assault sustained injuries as seen in Table 2 because those specific regions were targeted. Injuries due to mob justice were mainly to the limbs and head and neck regions, and are probably a consequence of high urban unemployment and increase in crime. Assaults caused injuries which were distributed to most body regions. 


\section{Duration from time of injury}

The duration from the time of injury to presentation to the A \& E Department of Mulago Hospital ranged from 0.5 to $14 \mathrm{hrs}$. The population's mean was 2.75 hours. Most of the patients presented to the hospital after 1 hour from the time of injury. Of the patients who presented within 1 hour of injury, $61.1 \%$ were in grade IV shock. This late hospital presentation is also observed in other studies $[17,18]$. This is likely to be due to: a long distance from hospital; lack of an organised pre-hospital care system which would facilitate early transfer of patients to the hospital; and poor communication due to lack of toll free functional emergency numbers.

Early presentation to hospital following injury has been known to improve survival. However, in this study, patients who presented early were more likely to die than those who presented late; a paradoxical observation. This can be explained by the injury characteristics of those who presented early; most had severe injuries and were in grade IV shock. They were most likely in a state of irreversible shock, a virtually certain prelude to death. Despite these findings, the benefits of early hospital presentation should not be disputed and efforts should be made to ensure that patients reach hospital early.

\section{Nature of injury sustained}

Scalp lacerations were the leading cause of shock (29.1\%), followed by ruptured spleen (20\%) and long bone fractures (12.7\%). The scalp is well vascularised and its vessels do not readily constrict when injured unlike vessels elsewhere in the body. This therefore promotes copious bleeding. Control of haemorrhage from scalp lacerations was achieved by pressure dressing and wound suturing, giving these patients a better prognosis. The spleen is a well vascularised organ whose injury can lead to significant blood loss and shock. Unlike scalp lacerations, bleeding from splenic injuries could not be controlled by pressure. Nearly all these patients had laparotomy to control bleeding because they failed to respond to fluid resuscitation.

Up to $2 \mathrm{~L}$ of blood can be lost from fracture of the femur and this is more likely to cause shock especially when bilateral. Bleeding from open fractures reduced after applying compression dressing and splinting the limb. Shock was also caused by other injuries as seen in Figure 4.

\section{Grade of shock}

Patients who presented with grade III shock (54.6\%) outnumbered those with grade IV shock (45.5\%). Of all the patients who were in grade III shock, 76.7\% presented to the A \& E Department after $1 \mathrm{hr}$ from the time of injury and many of them had scalp lacerations (43.3\%). The observation of patients in grade III shock being seen more is ostensibly because some of the patients in grade IV shock may have died before reaching hospital. Grade III shock patients were more likely to be in the progressive stage of shock, while those in grade IV shock could have entered the irreversible stage of shock. The body`s compensatory mechanisms were still able to keep these patients alive despite late presentation to hospital unlike those in grade IV shock who could have decompensated. Nearly all the patients in grade III shock responded well to intravascular fluid replacement following haemorrhage control. Their outcome was therefore good with most patients surviving the first 24 hour period.

\section{Medical care before and after admission to Mulago Hospital}

Only $8(14.6 \%)$ patients received pre-hospital care in the form of intravenous fluids, blood transfusion and medication from either clinics or other hospitals. Medical care given from the clinics was in the form of what is loosely termed as 'first aid. The observed fluid and blood requirements of the patients who had received pre-admission care were not much different from those who did not receive care. Statistical significance testing was not applicable given the low numbers of patients who received pre-hospital care. The type of fluids and the amount given was difficult to ascertain. This makes it further difficult to draw conclusions.

At Mulago Hospital all patients received intravenous fluids (NS or RL). Thirty seven (67.3\%) needed blood transfusion, while 18 (32.7\%) did not. The patients who did not need blood transfusion responded to the fluid bolus following haemorrhage control. These were all in grade III shock. Of those who needed blood transfusion, 7 did not receive it because blood was not available in the blood bank at the time. They all died. Blood transfusion was needed for all patients in grade IV shock. This shows that some patients with grade III shock can improve without blood transfusion. In a setting where blood is limited, early detection of shock and use of alternative fluids improves survival and decreases the need for blood transfusion.

Twenty seven $(40.1 \%)$ of the patients needed major surgery to control bleeding, and all the patients who were operated within the first 2 hours of admission survived. Those who were operated after 2 hours were more likely to have a poor outcome compared to those who were operated within 2 hours. This could have been due to complications of prolonged haemorrhagic shock such as acidosis, hypothermia and coagulopathy in these patients. They were also exposed to the risks of surgery and anaesthesia per se. Delays in surgery can be attributed to lack of adequate theatre space; the 2 available operation suites are used for all emergencies. Also inadequate manpower, lack of blood and theatre sundries, and a slow sterilisation process play a role in causing delays.

\section{Baseline $\mathrm{Hb}$ and $\mathrm{PCV}$}

There was no significant difference in mean $\mathrm{Hb}$ and PCV between survivors and non-survivors ( $P=0.275$ and 0.575 respectively). Blood transfusion was based on the clinical presentation of the patients and their response to the fluid bolus but not the $\mathrm{Hb}$ and PCV values. This was because $\mathrm{Hb}$ and PCV values could not be relied on in acute bleeding. These values tended to be high even when the patients were clinically anaemic. $\mathrm{Hb}$ and PCV are known to be high in acute bleeding due to haemoconcentration. Patients who presented with low $\mathrm{Hb}$ at admission could probably either have had massive haemorrhage or moderate haemorrhage with dilutional anaemia due to overzealous intravenous fluid resuscitation.

\section{Admission lactate levels}

Patients who sustained injuries due to assault, RTC and mob justice had higher mean admission serum lactate levels compared to the ones who had other mechanisms of injury (Table 6). Assaults, RTC and mob justice tend to cause multiple and extensive tissue injuries; a random pattern of injury. Multiple injuries provide multiple sites for blood loss and therefore more severe shock. This could explain why these patients had higher mean serum lactate levels. GSIs can cause multiple and extensive injuries. However, in our study patients who sustained injuries by this mechanism had single bullet injuries which were not so extensive.

Although patients who were assaulted had the highest mean serum lactate levels, more deaths were observed in patients with RTIs. This is probably because RTIs were the leading form of injuries, meaning that more patients were injured by this mechanism than the rest of other 
mechanisms. This translated to more deaths. The mechanism of injury for RTIs also probably has the highest energy transfer (deceleration/ acceleration effect) involved when compared to other injuries. Assault patients tended to have multiple injuries and therefore presented with the highest serum lactate levels, though not more deaths.

All these raised lactate levels apparently have clinical significance, though no statistical significance. A study with a larger sample size and longer duration could possibly bring out a statistically significant trend.

\section{Treatment outcome}

The $29.1 \%$ mortality of the study is lower than that reported by Kirya et al. [2] and Heckbert et al. [19], but higher compared to the study by Hill et al. [15]. Most of the deaths occurred within 1 hour of admission to Mulago Hospital, a finding consistent with that of another study $[20,21]$. Of all the patients who died, only 1 was in grade III and the rest were in grade IV shock. The patient in grade III shock died post operatively following a laparotomy for a ruptured spleen. She was also immunocompromised as a result of HIV infection and had surgery done after 2 hours of admission. These factors could have contributed to her death.

Grade IV shock is the severest form of shock and contributed to the highest mortality. These patients were most likely in the irreversible stage of shock, with severe tissue hypoxia. Prolonged hypoxia damages ATP generation mechanisms, intracellular organelles and cell membrane integrity leading to permanent cell damage and failure to respond to resuscitation. However, death due to reperfusion injury following intravenous fluid resuscitation cannot be ruled out. This occurs as circulation improves and is due to release of free radicals and cytokines from damaged tissues. These cause further tissue damage and death.

Strict adherence to the ATLS guidelines, and the injury characteristics of the study population could have been responsible for the relatively low mortality in this study. Most of the injuries sustained were to the head and neck region, and limbs. A majority of these injuries were amenable to haemorrhage control by external compression using digital pressure, pressure dressing and wound suturing, unlike torso injuries. However, this mortality prevalence is still high compared to that in high income country studies.

\section{Distribution of factors which are known to affect outcome}

There were 3 factors that significantly affected survival in this study, namely: i) the mechanism of injury, ii) pre-Mulago Hospital care given, and iii) the age of the patient. The Odds Ratio and $\mathrm{P}$ values for the need for blood transfusion and time to surgery were not applicable since one of the categories had no mortality. Patients who presented within one hour of injury were more likely to die than those who presented after one hour, OR=0.23 (95\% CI 0.07-0.80) and $P=0.021$. Other studies have reported reduced mortality in patients who present within one hour of injury ('the Golden hour') [22,23]. This difference could be explained by the injury characteristics of these patients and their inhospital management. One patient had a penetrating chest injury with massive haemothorax and suspected cardiac injury, 1 had liver laceration and 2 had vascular injuries. These were severe injuries with massive haemorrhage. All these patients received blood in the range of 1 to 5 units (average, 2.75 units), but died despite early presentation. Other patients had injuries like ruptured spleen and lacerations which normally would improve well with appropriate surgery and resuscitation, but died because they did not receive a blood transfusion which was essential at that time.
Of the 18 patients who presented within 1 hour of injury, 11(61.1\%) were in grade IV shock, while only 14 of the 37 (37.8\%) patients who presented after 1 hour were in grade IV shock. Of all the patients who presented with grade IV shock within 1 hour, $66.7 \%$ needed major surgery to control bleeding and $70 \%$ of them had surgery done after 2 hours. Only $40.5 \%$ of the patients who presented with grade IV shock after 1 hour from injury needed major surgery to control bleeding and $81.8 \%$ of them had surgery done after 2 hours. This stresses the point that patients who presented early had severe injuries, and that most of the patients who needed surgery to control bleeding did not have it done early. Delay in accessing surgery was mainly due to Operation Theatre constraints. This could have contributed to the mortality seen in these patients.

Patients in grade IV shock were more likely to die than those in grade III shock, OR $=43.50(95 \%$ CI $5.08-372.71)$ and $P=0.001$. This is because haemorrhagic shock progresses from grade I to IV if not reversed [16]. Grade IV shock is the severest form of shock and these patient eventually develop complications of uncontrolled haemorrhage in shock-acidosis, coagulopathy and hypothermia, which constitute the lethal triad of death. Patients in grade IV shock were more likely to enter the irreversible stage of shock and not respond to treatment. Of all the patients in grade IV shock, $88 \%$ of them needed major surgery to control bleeding. This shows that these patients sustained severe injuries and were at a high risk of death.

Patients who needed major surgery to control bleeding were also more likely to die than those who did not, OR 14 (95\% CI 2.76-71.04) and $\mathrm{p}=0.001$. These patients were generally more seriously injured than those not requiring major surgery. They therefore were at an increased risk of death in the first place. In addition, they were also exposed to the risks of surgery per se compared to the ones who did not need major surgery.

The mechanism of injury, age of the patients and pre-Mulago Hospital care distribution were not significant factors in outcome. Those who received pre-hospital care did not have a significant reduction in mortality $(p=0.286)$. This was most likely due to inadequate and/or inappropriate care given in the pre-hospital setting. What comes out clearly here is the need for quality pre-hospital care in order to reduce mortality. The statistical insignificance of the mechanism of injury as a factor of outcome can be explained by the nature of our study. We monitored patients for 24 hours only. Longer periods of patient follow up (48 hours to 7 days) could possibly have resulted in significant differences among the various mechanisms of injury. Age of the patients was also not significant due to similarity in the age distribution between survivors and non-survivors. This possibly could have been significant if elderly patients were present in the study.

\section{Adequacy of resuscitation}

At admission, all patients had serum lactate levels $>2.2 \mathrm{mmol} / \mathrm{L}$ (average lactate levels at admission was $7.24 \mathrm{mmol} / \mathrm{L}$ ). The serum lactate of the non-survivors ranged from 4.66 to $20.71 \mathrm{mmol} / \mathrm{L}$, with a mean of $9.65 \mathrm{mmol} / \mathrm{L}$. After management in the first 24 hours, $66.7 \%$ of the survivors had a reduction in the serum lactate levels to $<2.2 \mathrm{mmol} / \mathrm{L}$, an indication of adequate resuscitation. Serum lactate was still $>2.2$ $\mathrm{mmol} / \mathrm{L}$ in $33.3 \%$ of the patients who survived, indicating inadequate resuscitation.

Lactate measurement has been used to detect tissue hypoperfusion and to monitor the resuscitation of patients in shock [24]. Serum lactate reflects the amount of lactic acid in the blood and is a fairly sensitive and reliable indicator of tissue hypoperfusion and hypoxia [21]. The 
Citation: Elaju M, Ongom PA, Kijjambu SC, Wangoda R, Makobore P (2013) Short Term Outcome of Haemorrhagic Shock in Trauma at Mulago Hospital, an Urban Tertiary Hospital in Sub Saharan Africa. Trop Med Surg 1: 148. doi:10.4172/2329-9088.1000148

return of serum lactate levels to normal within 24 hours in trauma patients following resuscitation has been shown to improve survival [25]. In relation to this is the observation by Krishna et al. that described interventions that decrease lactate levels values to normal, early in resuscitation, may improve chances of survival [25]. It was observed that such interventions ought to be considered effective therapy in patients with haemorrhagic shock. Based on lactate levels, one third (33.3\%) of the survivors were inadequately resuscitated at 24 hours. These patients were therefore at risk of developing multiple system dysfunction and death later due to ongoing occult tissue hypoxia. Abramson et al. described an increase in the chances of mortality with delayed return of lactate levels to normal [24]. This entails that follow up of patients for $48 \mathrm{hrs}$ and above may give a better prognostic evaluation for these patients, especially the critically injured [25].

The systolic BP was normal in $89.7 \%$ of the patients after $24 \mathrm{hrs}$, while it was $>140 \mathrm{mmHg}$ in $10.0 \%$ of the patients. However, the 24 hours serum lactate of the patients with elevated BP was normal. The elevation in systolic BP could have been due to: overzealous resuscitation (IV fluids), pain, anxiety or true hypertension which the patients were unaware of Considering other vital signs, 35.9\% still had tachycardia and $48.7 \%$ were tachypnoeic. Following adequate resuscitation the vital signs should normalise. Persistence of tachycardia and tachypnoea could mean inadequate resuscitation, anxiety or pain. Standardisation of analgesia was difficult due to stock-outs in the A \& E Department. Urine output was above $30 \mathrm{~mL} / \mathrm{hr}$ in most patients indicating good renal perfusion.

During the study there were a few prominent shortcomings. Though it was an observational study, active participation by the investigators could not be avoided. This was chiefly due to ethical reasons. This intervention can compromise the outcome of treatment. We also based our vital signs measurement on systolic BP measurement. This alone does not detect occult (compensated) shock at admission, leaving only grade III and IV shock to be studied. We did not look at grade I and II shock. Other biochemical markers such as base deficit and arterial blood gases which we would have used to monitor resuscitation were not measured.

\section{Conclusions}

The $24 \mathrm{hr}$ mortality due to haemorhagic shock in Mulago Hospital (29.1\%) is high compared to that in centres in high income countries. Of the factors known to affect mortality in patients with traumatic haemorrhagic shock, grade IV shock and the need for major surgery to control bleeding significantly increased mortality at Mulago Hospital. Time since injury had a negative correlation with mortality, while the mechanism of injury, age of the patients and pre-hospital care provided, did not affect mortality.

Blood pressure measurement alone is not a reliable method for determining the adequacy of resuscitation. The use of serum lactate to monitor resuscitation can identify inadequately resuscitated patients.

It is important that strategies to reduce mortality are put in place, pre-hospital and in-hospital, involving injury prevention and control, and patient management. Serum lactate levels should be routinely used alongside vital signs to monitor resuscitation in trauma patients. Lactate levels need to be followed for longer periods of time in critical patients.

\section{Authors' Contributions}

ME conceptualized the theme, collected and analysed the data, and wrote the manuscript. PAO designed and co-wrote the manuscript.
SCK edited the manuscript. PAO, SCK, RW and PM monitored the study. All authors read and approved the final manuscript.

\section{Acknowledgement}

The authors wish to extend their sincere gratitude to the staff of $A$ \& $E$ Department, Mulago National Referral and Teaching Hospital and the Department of Surgery, School of Medicine, Makerere University College of Health Sciences for the material support and their cooperation.

\section{References}

1. Penden M (2004) World Report on Road Traffic Injury Prevention. World Health Organization, Geneva 1-66.

2. Kirya F, Kijjambu S, Ezati I (2002) Outcome of major trauma at Mulago Hospital in Uganda: assessment using the TRISS methodology. East and Centra African Journal of Surgery 7: 59-62.

3. Demetriades D, Murray J, Charalambides K, Alo K, Velmahos G, et al. (2004) Trauma fatalities: time and location of hospital deaths. J Am Coll Surg 198: 20-26.

4. Cherkas $D$ (2011) Traumatic hemorrhagic shock: advances in fluid management Emerg Med Pract 13: 1-19.

5. Krug EG, Sharma GK, Lozano R (2000) The global burden of injuries. Am J Public Health 90: 523-526.

6. Murray CJ, Lopez AD (1997) Mortality by cause for eight regions of the world Global Burden of Disease Study. Lancet 349: 1269-1276.

7. Hsia RY, Ozgediz D, Mutto M, Jayaraman S, Kyamanywa P, et al. (2010) Epidemiology of injuries presenting to the national hospital in Kampala, Uganda: implications for research and policy. Int J Emerg Med 3: 165-172.

8. Jayaraman S, Ozgediz D, Miyamoto J, Caldwell N, Lipnick MS, et al. (2011) Disparities in injury mortality between Uganda and the United States: comparative analysis of a neglected disease. World J Surg 35: 505-511.

9. Gutierrez G, Reines HD, Wulf-Gutierrez ME (2004) Clinical review: hemorrhagic shock. Crit Care 8: 373-381.

10. Krausz MM (2006) Initial resuscitation of hemorrhagic shock. World J Emerg Surg 1: 14.

11. Garrioch MA (2004) The body's response to blood loss. Vox Sang 87 Suppl1: 74-76.

12. Chalya PL, Mabula JB, Dass RM, Mbelenge N, Ngayomela IH, et al. (2012) Injury characteristics and outcome of road traffic crash victims at Bugando Medical Centre in Northwestern Tanzania. J Trauma Manag Outcomes 6: 1.

13. Bonanno FG (2012) Hemorrhagic shock: The "physiology approach". J Emerg Trauma Shock 5: 285-295.

14. Choi Y, Ametepi P (2013) Comparison of medicine availability measurements at health facilities: evidence from Service Provision Assessment surveys in five sub-Saharan African countries. BMC Health Serv Res 13: 266.

15. Hill DA, West RH, Roncal S (1995) Outcome of patients with haemorrhagic shock: an indicator of performance in a trauma centre. J R Coll Surg Edinb 40: $221-224$

16. Committee on Trauma, American College of Surgeons (2004) Advanced Tauma Life Support. (7thedn). Chicago, Ch 3: 69-85.

17. Mujuni E, Wangoda R, Ongom P, Galukande M (2012) Acute traumatic coagulopathy among major trauma patients in an urban tertiary hospital in sub Saharan Africa. BMC Emerg Med 12: 16.

18. Kobusingye OC, Lett RR (2000) Hospital-based trauma registries in Uganda. J Trauma 48: 498-502.

19. Heckbert SR, Vedder NB, Hoffman W, Winn RK, Hudson LD, et al. (1998) Outcome after hemorrhagic shock in trauma patients. J Trauma 45: 545-549.

20. Antinone R, Kress T (2009) Measuring serum lactate. Nursing Critical Care 4: 56.

21. Hai SA (2004) Permissive hypotensive resuscitation--an evolving concept in trauma. J Pak Med Assoc 54: 434-436.

22. Sogut O, Sayhan MB, Gokdemir MT, Boleken ME, Al B, et al (2011) Analysis of 
Citation: Elaju M, Ongom PA, Kijjambu SC, Wangoda R, Makobore P (2013) Short Term Outcome of Haemorrhagic Shock in Trauma at Mulago Hospital, an Urban Tertiary Hospital in Sub Saharan Africa. Trop Med Surg 1: 148. doi:10.4172/2329-9088.1000148

Page 10 of 10

hospital mortality and epidemiology in trauma patients: a multi-centre study. $\mathrm{J}$ Curr Surg 1: 19-24

23. Cocchi MN, Kimlin E, Walsh M, Donnino MW (2007) Identification and resuscitation of the trauma patient in shock. Emerg Med Clin North Am 25: 623-642, vii.
24. Abramson D, Scalea TM, Hitchcock R, Trooskin SZ, Henry SM, et al. (1993) Lactate clearance and survival following injury. J Trauma 35: 584-588.

25. Krishna U, Joshi SP, Modh M (2009) An evaluation of serial blood lactate measurement as an early predictor of shock and its outcome in patients of trauma or sepsis. Indian J Crit Care Med 13: 66-73. 